\title{
STUDY OF KNOWN RISK FACTORS IN BREAST CANCER PATIENTS IN THE REGIONAL POPULATION
}

\author{
Ashok Kumar1, Abhilash Bipin Kumar Tirkey², Sumitoj Singh³, Rajiv Devgan ${ }^{4}$ \\ ${ }^{1}$ Assistant Professor, Department of Surgery, Guru Nanak Dev Hospital, Amritsar, Punjab, India. \\ 2Junior Resident, Department of Surgery, Guru Nanak Dev Hospital, Amritsar, Punjab, India. \\ 3 Professor, Department of Surgery, Guru Nanak Dev Hospital, Amritsar, Punjab, India. \\ 4 Professor and HOD, Department of Radiotherapy, Guru Nanak Dev Hospital, Amritsar, Punjab, India.
}

\section{ABSTRACT}

\section{BACKGROUND}

Breast cancer was the most common cancer in India in 2018. It also caused the most cancer related deaths in India in 2018. In order to decrease the mortality from breast cancer, it is essential to identify the population at risk, and diagnose breast cancer at an early stage. However, breast cancer risk factors have been studied mostly in European and American countries whose traditions, practices, habits and lifestyles are enormously different. Even within a country like India there is vast diversity with respect to various cultures, traditions and practices. Hence the risk factors for all these diverse groups may not be the same. We wanted to study the known risk factors in breast cancer patients in the regional population.

\section{METHODS}

This is a cross sectional study of 100 women with a histopathological diagnosis of breast cancer who presented to Government Medical College, Amritsar.

\section{RESULTS}

Breast cancer presented at an earlier age $52.02 \pm 9.42$ and at a later stage compared to other developed countries. $40 \%$ of the patients had no risk factors for breast cancer. The most common risk factor was BMI $\geq 25$ in $40 \%$ cases. Life style related risk factors- alcohol and smoking were present in 4\% cases. Risk factors related to estrogen exposure were early menarche (1\%), nulliparity (1\%), lack of breast feeding (0\%), OCP intake (6\%), late menopause (4\%), HRT (0\%). Family history of breast cancer was present in $1 \%$. None of the cases had significant radiation exposure prior to diagnosis.

\section{CONCLUSIONS}

The risk factor profile for breast cancer in the Indian population differs from that of other countries in whom the risk factors were first identified and studied. Hence a large multicentre study is required to identify the risk factors relevant to the Indian population.

HOW TO CITE THIS ARTICLE: Kumar A, Tirkey ABK, Singh S, et al. Study of known risk factors in breast cancer patients in the regional population. J. Evolution Med. Dent. Sci. 2019;8(18):1446-1450, DOI: 10.14260/jemds/2019/322

\section{BACKGROUND \\ In 2018, breast cancer has been the most common cancer} across all ages and both sexes with 1,62,468 new cases reported in the year. It has the highest 5 -year prevalence among all cancers in India which is 4,05,456 cases. It has also been the most common cause of cancer related death with a mortality of 87,090 cases in $2018 .{ }^{1}$ Hence breast cancer is an alarming nationwide health problem of an ominous magnitude causing great morbidity and mortality.

In spite of the magnitude of this problem, there is no national screening programme for breast cancer. ${ }^{2}$ the mean age of presentation of breast cancer in the United States of America is 62 years whereas in India it is 50 years of age..$^{3,4} \mathrm{~A}$ vast proportion of the population still lives in the rural area and are uneducated. They tend to present late with the disease due to inaccessible health care, cost, lack of knowledge or social stigma. ${ }^{5}$ Even in the urban population, practices like self-breast examination are uncommon.

'Financial or Other Competing Interest': None.

Submission 16-03-2019, Peer Review 20-04-2019,

Acceptance 26-04-2019, Published 06-05-2019.

Corresponding Author:

Dr. Sumitoj Singh,

C-12, Medical College Campus,

Government Medical College,

Amritsar-143001, Punjab, India.

E-mail: sumitoj@rediffmail.com

DOI: $10.14260 /$ jemds/2019/322
This leads to late presentation where the treatment may not be as successful as early breast cancer.

It is important to identify the risk factors present among individuals. This will enable early screening and diagnosis among this population which is at an increased risk for breast cancer. The risk factors commonly implicated for breast cancer are factors related to oestrogen exposure- early menarche ( $\leq 11$ years), nulliparity, lack of breast feeding, intake of oral contraceptive pills, late menarche ( $\geq 55$ years), use of hormone replacement therapy. Life style related risk factors are - smoking, alcohol, night shift work, BMI $\geq 25$ and lack of physical activity. Family history of breast cancer and exposure to therapeutic radiation are also important risk factors for breast cancer.

India is a country with vast differences in cultures and practices within the country itself and from the Western civilization. Hence the risk factors for breast cancer may not be the same as for them. This study aims to find the prevalence of these known risk factors among breast cancer patients in the regional population.

\section{METHODS}

This is a cross sectional study conducted in Government Medical College, Amritsar where 100 females with histopathological diagnosis of breast cancer were selected by convenience sampling. Any female patient with breast cancer who consented for the study was eligible for selection. 
A questionnaire based one on one interview was undertaken where the following information was obtained. Demographic variables obtained were age, educational status, occupation and marital status. BMI was calculated using height and weight of the patient. Questions related to breast cancer risk factors were asked about age of menarche, number of children, age at first child birth, breast feeding, intake of oral contraceptive pills, age at menopause, use of hormone replacement therapy. Questions about lifestyle related risk factors like night shift work, lack of physical activity, smoking and alcohol intake were also asked. Questions related to family history included any first degree or second degree relative with breast cancer in the family. Information about any exposure to therapeutic radiation was also obtained in the questionnaire. Other disease related information obtained were stage at diagnosis, histological diagnosis, ER/PR and Her-2/neu receptor status.

The data obtained was entered into excel sheet, and analysis was done with the help of excel functions and is presented here as mean and percentages. Since the risk factor assessment is based on the verbal responses that involve recalling information of several years back, the possibility of memory recall bias cannot be ruled out.

The study was carried out between January 2017 and July 2018 after approval from the institutional ethics committee.

\section{RESULTS}

The mean age at presentation was $52 \pm 9.42$ years with $12 \%$ presenting between $30-40$ years of age, $22 \%$ between $41-50$, $56 \%$ between $51-60,8 \%$ between $61-70$ and $2 \%$ cases $>70$ years of age. The youngest case was 32 years old and the oldest case was 85 years old.

$20 \%$ of the cases were illiterate, $17 \%$ had completed primary education, $24 \%$ had completed middle school education, $25 \%$ had completed matric and $14 \%$ had studied beyond matric with only $1 \%$ having completed postgraduation. $88 \%$ of the cases were housewives, $4 \%$ were teachers and labourers each, $2 \%$ were clerks, $1 \%$ had a government job and $1 \%$ had tailoring as her occupation. None of the cases were in occupations involving night-shift work.

The mean BMI of the patients was $24.85 \pm 2.98 .60 \%$ of the cases had BMI $<25,35 \%$ had BMI between 25-29.9 and 5 $\%$ cases had BMI $\geq 30.22 \%$ of cases lacked exercise or moderate physical activity in their daily routine. $4 \%$ of the cases, labourers by occupation had a history of consumption of alcohol and smoking whereas $96 \%$ cases had never smoked or consumed alcohol.

$21 \%$ of the cases had presented at stage IIa, $32 \%$ at IIb, $28 \%$ at IIIa, $17 \%$ at IIIb and $2 \%$ at stage IV of the disease. None of the cases presented at stage I. Most common histological sub type was infiltrating ductal carcinoma (84\%). Other histological subtypes were infiltrating lobular carcinoma (5\%), papillary carcinoma (9\%), medullary and micro-papillary, each $1 \%$.

The mean age at menarche was $14.23 \pm 1.21$. Only one case $(1 \%)$ had menarche at 11 years of age. $88 \%$ had menarche between $12-14$ years and $11 \%$ between $15-18$ years. All the cases in the study were married before 30 years of age. $13 \%$ of the cases were married at $\leq 18$ years, $37 \%$ between $19-21,36 \%$ between $22-24$ and $14 \%$ between $25-$ 30 . The mean age at marriage was $21.59 \pm 2.64$. Only one case
(1\%) was nulliparous, $2 \%$ had one child, $41 \%$ had 2 children, $33 \%$ had 3 children and $23 \%$ had 4 children.
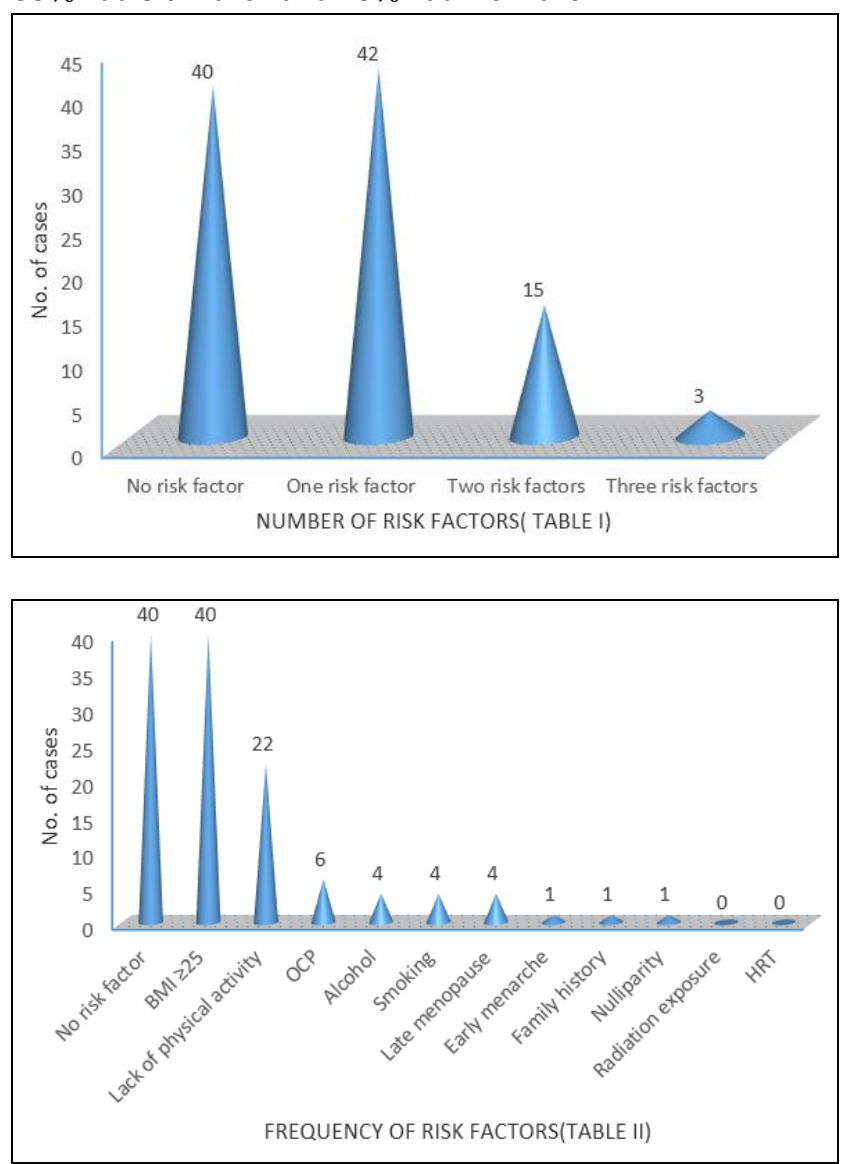

All the children were born before 30 years of age. All of the children have been breastfed.

Among the cases only $6 \%$ had used oral contraceptive pills but none had taken it for more than 6 months. 94\% cases had never used oral contraceptive pills. $18 \%$ of the cases were pre-menopausal and $82 \%$ of the cases were postmenopausal. $11 \%$ cases attained menopause between $40-45$ years of age, $67 \%$ between $46-50$ and $4 \%$ after 50 years of age. None of cases had ever taken hormone replacement therapy.

None of the cases had been exposed to therapeutic radiation. Only one case (1\%) had a first degree relative (mother) with breast cancer. None of the other cases had a first or second degree relative with breast cancer.

$40 \%$ of the cases had none of the known risk factors for breast cancer. $42 \%$ cases had one risk factor, $15 \%$ had 2 risk factors and $3 \%$ had 3 risk factors (Table I). The summary of distribution of risk factors is seen in Table II.

\section{DISCUSSION}

Our study was conducted at Guru Nanak Dev Hospital, Amritsar. 100 Female patients with a diagnosis of breast cancer chosen for the study. $20 \%$ of the cases were illiterate and only $39 \%$ were educated up to matric or higher one of whom had completed post-graduation. $88 \%$ of the cases were housewives.

None of the cases in the study presented to the hospital at stage I of the disease. 53\% were diagnosed at stage II and $45 \%$ were diagnosed at stage III. $2 \%$ cases had metastatic stage 4 disease at the time of presentation. 
In our study, the youngest case was 32 years old and the oldest case was 85 years old. Mean age of presentation was 52.02 years with a standard deviation of 9.42 years. This finding is close to the mean age 50.1 with a standard deviation of 10.5 years found in a study done by Brinder Chopra et al. This age is much lesser compared to the United States of America where the mean age of presentation is 62 years.

In our study, mean BMI was 24.85 with a standard deviation of 2.98 with 40 percent of the cases had BMI above 25. A study by Tomi Akinyemiju et al had $43 \%$ cases with BMI above 25.6

Mean age at menarche was $14.23 \pm 1.21$ years with only one case having menarche at 11 years and none earlier than that. In a study conducted in Mumbai by Dyavarishetty, 3.3\% cases had their menarche before 12 years of age. ${ }^{7}$ In a study from Udipi, 31\% cases had their menarche before 12 years of age. ${ }^{8}$ In a meta-analysis by collaborative group on hormonal factors in breast cancer, mean age at menarche was 13.1 years (SD 1.7) where 16\% (49464) of the controls reported menarche at age 11 years or younger. ${ }^{9}$

All the cases in our study were married. Only one case (1\%) was nulliparous, $2 \%$ had one child and $97 \%$ cases were multiparous. In a study conducted in Mumbai by Dyavarishetty, $3 \%$ of the case were unmarried. $4.4 \%$ of the cases were had their first pregnancy after 30 years of age. In a study from Udipi, $56 \%$ of the cases had first pregnancy at 30 years or above. All the mothers had breastfed their child/ children in our study. In the Mumbai study, only $1.6 \%$ did not breast feed their child.

$6 \%$ of cases in our study had taken oral contraceptive pills nut none of them had taken it for more than 6 months. In the Mumbai study, $2.8 \%$ cases had taken oral contraceptive pills. Similar finding was seen in the Udipi study.

$18 \%$ of the cases in our study were pre-menopausal and $82 \%$ were post-menopausal. Mean age at menopause was $46.82 \pm 2.99$ years. This age was lower than the mean age found in the meta-analysis by collaborative group on hormonal factors in breast cancer, in which mean age at natural menopause was $49 \cdot 3$ years (SD 4.6), with 15\% (26285 of 170413) reporting menopause before age 45 years, 75\% (127 984) between the ages 45 and 54 years, and $10 \%(16144)$ at age 55 years or older. The RR of breast cancer increased by a factor of 1.029 (1.025-1.032, $\mathrm{p}<0.0001$ ) for every year older at menopause in the study. $4 \%$ of the cases in our study had attained menopause after 50 years of age.

In a study published in the New England Journal of Medicine, the relative risk of breast cancer among all current and recent users of hormonal contraception was 1.20 (95\% confidence interval [CI], 1.14 to 1.26). This risk increased from 1.09 (95\% CI, 0.96 to 1.23 ) with less than 1 year of use to 1.38 (95\% CI, 1.26 to 1.51 ) with more than 10 years of use $(\mathrm{P}=0.002) .{ }^{10} \mathrm{~A}$ more recent study in the JAMA Oncology found no association between Oral Contraceptive use and breast cancer. ${ }^{11}$ In our study, $94 \%$ of the cases had never taken any oral contraceptive pills. Only $6 \%$ of the cases had ever taken oral contraceptives at least once in their life time. None of the cases were taking oral contraceptive pills within 1 year of diagnosis of breast cancer and none of the cases had taken oral contraceptive pills for more than 6 months in their life time. In the Mumbai study, $2.8 \%$ cases had consumed oral contraceptive pills and only 2 of them had consumed it for more than 5 years. A similar finding was seen in the Udipi study.

In a register-based cohort study in Norway, The hazard ratios associated with prescriptions of hormone replacement therapy for more than 1 year were 2.06 (1.90-2.24) for oestrogen + progesterone combinations and 1.03 (0.85-1.25) for systemic oestrogens. Invasive lobular carcinoma was more strongly associated with use of oestrogen and progesterone combinations, $\mathrm{HR}=3.10$ (2.51-3.81), than nonlobular carcinoma, HR $=1.94(1.78-2.12) \cdot{ }^{12}$ In a study by Jones ME et. al, the hazard ratio in relation to current oestrogen plus progesterone hormone replacement therapy use relative to those with no previous use was $2.74(95 \%$ confidence interval (CI): 2.05-3.65) for a median duration of 5.4 years of current use, reaching 3.27 (95\% CI: 1.53-6.99) at $15+$ years of use. The Hazard Ratio for oestrogen-only HRT was not increased (HR=1.00; 95\% CI: 0.66-1.54). ${ }^{13}$ In our study, none of the patients had ever taken menopausal hormone replacement therapy.

In a study among patients of Hodgkin's Lymphoma who underwent chest radiotherapy, the pooled relative risk of secondary breast cancer was 8.23 (95\% CI, 5.43-12.47, $\mathrm{I}^{2}=96 \%$ ). The relative risk was found inversely related to age at diagnosis of HL with the highest rate $(68.7$; [95 \% CI, 28.08-168.11], $\mathrm{I}^{2}=79 \%$ ), occurred in young patients ( $\leq$ 15 years old), whereas the RR in older women $\geq 40$ years old was not significant ( 0.55 ; [95\% CI, 0.09-3.52]). Analysis of the effect of treatment modalities showed that the RR rates were (4.70; [95\% CI, 3.28-6.75], $\mathrm{I}^{2}=74 \%$ ), (5.65; [95\%CI, 2.9410.88] for radiotherapy and radiotherapy + chemotherapy respectively. ${ }^{14}$ In our study, none of the cases had significant radiation exposure prior to their diagnosis.

Khazrami et al found that having family history of single (HR 1.8; $95 \%$ CI 1.8-1.9) or multiple (HR 2.7; $95 \%$ CI 2.6-2.9) relatives with breast cancer was associated with higher risk of breast cancer. ${ }^{15}$ In a study published in the JAMA Internal Medicine, first-degree family history was associated with an increased risk of breast cancer among women ages 65 to 74 years (hazard ratio [HR],1.48; 95\% CI, 1.35-1.61) and 75 years and older (HR, 1.44; 95\% CI, 1.28-1.62).16 In our study, only one case which is $1 \%$, diagnosed at 43 years of age, had a first degree relative, her mother, with breast cancer. None of the cases had any second-degree relative suffering from breast or ovarian cancer. In the Mumbai study, $0.7 \%$ of the cases had a positive family history. In the Udipi study, none of the cases had a positive family history. In a study in Kerala, $4.7 \%$ of the cases had a family history of breast cancer. ${ }^{17}$ Although only 1 case had a positive family history, 34\% of the cases were diagnosed at $<50$ years of age. Weitzel JN et al had found that patients diagnosed with breast or ovarian cancer at age $<50$ years with a limited family history may have an underestimated probability of BRCA1/2 mutations based on family history alone. ${ }^{18}$

A meta-analysis by Key J et al that combined the results of 98 studies found excess risk associated with alcohol drinking was 22\% (95\% CI: 9-37\%); each additional $10 \mathrm{~g}$ ethanol/day was associated with risk higher by $10 \%$ (95\% CI: 5-15\%). ${ }^{19}$ In our study only $4 \%$ cases, labourers by occupation, consumed alcohol. A comprehensive meta-analysis by Bagnardi V. et al found a relative risk of 1.61 for breast cancer with a clear dose- risk response. ${ }^{20}$ Choi YJ et al found that 
even very light ( $\leq 0.5$ drink/day) drinking, led to an increased risk for breast cancer. ${ }^{21} 96 \%$ of the cases in our study denied ever having consumed alcohol.

A large EPIC cohort study found that when compared to women never exposed to smoking, current, former and currently exposed passive smokers were at increased risk of breast cancer (hazard ratios (HR) [95\% confidence interval (CI)] 1.16 [1.05-1.28], 1.14 [1.04-1.25] and 1.10 [1.01-1.20]), respectively. ${ }^{22}$ In our study only $4 \%$ of the cases had a history of smoking. $96 \%$ of the cases had never consumed or smoked tobacco in any form. In the generations cohort study by Jone ME et al the hazard ratio (reference group was never smokers) was 1.14 (95\% CI 1.03-1.25; $\mathrm{P}=0.010)$ for ever smokers, 1.24 (95\% CI 1.08-1.43; $\mathrm{P}=0.002)$ for starting smoking at ages $<17$ years, and $1.23(1.07-1.41 ; \mathrm{P}=0.004)$ for starting smoking $1-4$ years after menarche. 23

In a meta-analysis of 139 prospective and retrospective studies by Hardefeldt PJ et al, physical activity significantly reduced the risk of breast cancer (odds ratio [OR], 0.78; 95\% confidence interval [CI], 0.76-0.81; $\mathrm{P}<.001$ ), with highintensity physical activity being slightly more protective (OR, $0.73 ; 95 \% \mathrm{CI}, 0.65-0.81 ; \mathrm{P}<.001)$ than low-intensity exercise (OR, 0.79; 95\% CI, 0.72-0.86; P <.001). The effect size for general exercise was similar in both premenopausal (OR, 0.79; 95\% CI, 0.73-0.87; $\mathrm{P}<.001$ ) and postmenopausal (OR, 0.82; 95\% CI, 0.78-0.86; $\mathrm{P}<.001$ ) women. Additionally, weight loss reduced the risk of breast cancer incidence (OR, 0.82; 95\% CI, 0.67-0.97). ${ }^{24}$ In our study, Regular physical activity was undertaken by $78 \%$ of the cases. Only $22 \%$ cases did not undertake physical activity of some kind in their daily routine.

It was interesting to note that $40 \%$ of the cases in our study did not have any of the known risk factors for breast cancer and $60 \%$ had at least one risk factor for breast cancer. $16 \%$ of the women in the Mumbai study had at least one known risk factor for breast cancer. In the Udipi study, 79\% cases had at least one risk factor for breast cancer.

Majority of the cases in our study had lifestyle related modifiable risk factors like high BMI and lack of physical activity. Other modifiable risk factors included smoking, alcohol and oral contraceptive pills. Only $7 \%$ of the cases had non modifiable risk factors which included family history and risk factors related to oestrogen exposure like early menarche, late menopause and nulliparity.

\section{CONCLUSIONS}

Hence, a large multi-centre study is needed to identify all known and possible risk factor of breast cancer relevant to the Indian population so that risk stratification can be used for earlier identification of patients with risk of breast cancer. This will help in earlier and more comprehensive treatment. This will be a stepping stone to the mammoth task of decreasing breast cancer mortality and morbidity which is currently the most common cancer in India.

\section{REFERENCES}

[1] Bray F, Ferlay J, Soerjomataram I, et al. Global cancer statistics 2018: GLOBOCAN estimates of incidence and mortality worldwide for 36 cancers in 185 countries. CA Cancer J Clin 2018;68(6):394-424.

[2] Gupta S. Breast cancer: Indian experience, data and evidence. South Asian J Cancer 2016;5(3):85-6.
[3] Noone AM, Howlader N, Krapcho M, et al. eds. SEER Cancer Statistics Review, 1975-2015. Table 1.12. Median age of cancer patients at diagnosis, 2011-2015. National Cancer Institute. Bethesda, MD. Updated September 10, 2018.

[4] Chopra B, Kaur V, Singh K, et al. Age shift: Breast cancer is occurring in younger age groups - Is it true? Clin Cancer Investig J 2014;3(6):526-9.

[5] Sathwara JA, Balasubramaniam G, Bobdey SC, et al. Sociodemographic factors and late-stage diagnosis of breast cancer in India: a hospital-based study. Indian J Med Paediatr Oncol 2017;38(3):277-81.

[6] Akinyemiju T, Wiener H, Pisu M. Cancer-related risk factors and incidence of major cancers by race, gender and region: analysis of the NIH-AARP diet and health study. BMC Cancer 2017;17(1):597.

[7] Dyavarishetty PV, Kowli SS. Prevalence of risk factors for breast cancer in women aged 30 years and above in Mumbai. Int J Community Med Public Health 2018;5(2):647-51.

[8] Shadap A. Profile picture of risk factors for breast cancer among community women of Udupi, Karnataka. NUJHS 2016;4(6):29-31.

[9] Collaborative Group on Hormonal Factors in Breast Cancer. Menarche, menopause and breast cancer risk: individual participant meta-analysis, including 118 964 women with breast cancer from 117 epidemiological studies. Lancet Oncol 2012;13(11):1141-51.

[10] Mørch LS, Skovlund CW, Hannaford PC, et al. Contemporary hormonal contraception and the risk of breast cancer. N Engl J Med 2017;377(23):2228-39.

[11] Michels KA, Pfeiffer RM, Brinton LA, et al. Modification of the associations between duration of oral contraceptive use and ovarian, endometrial, breast and colorectal cancers. JAMA Oncol 2018;4(4):516-21.

[12] Suhrke P, Zahl PH. Breast cancer incidence and menopausal hormone therapy in Norway from 2004 to 2009: a register-based cohort study. Cancer Med 2015;4(8):1303-8.

[13] Jones ME, Schoemaker MJ, Wright L, et al. Menopausal hormone therapy and breast cancer: What is the true size of the increased risk? $\mathrm{Br} \mathrm{J}$ Cancer 2016;115(5):607-15.

[14] Ibrahim EM, Abouelkhair KM, Kazkaz GA, et al. Risk of second breast cancer in female Hodgkin's lymphoma survivors: a meta-analysis. BMC Cancer 2012;12:197.

[15] Kharazmi E, Chen T, Narod $S$, et al. Effect of multiplicity, laterality and age at onset of breast cancer on familial risk of breast cancer: a nationwide prospective cohort study. Breast Cancer Res Treat 2014;144(1):185-92.

[16] Braithwaite D, Miglioretti DL, Zhu W, et al. Family history and breast cancer risk among older women in the breast cancer surveillance consortium cohort. JAMA Internal Medicine 2018;178(4):494-501.

[17] Augustine P, Jose R, Amrtithlal A, et al. Usefulness of Gail Model Breast Cancer Risk Assessment Tool in estimating the risk for development of breast cancer in women of Kerala, India. Academic Med J of India 2001;3(4):117-22. 
[18] Weitzel JN, Lagos VI, Cullinane CA, et al. Limited family structure and BRCA gene mutation status in single cases of breast cancer. JAMA 2007;297(23):2587-95.

[19] Key J, Hodgson S, Omar RZ, et al. Meta-analysis of studies of alcohol and breast cancer with consideration of the methodological issues. Cancer Causes \& Control 2006;17(6):759-70.

[20] Bagnardi V, Rota M, Botteri E, et al. Alcohol consumption and site-specific cancer risk: a comprehensive dose-response meta-analysis. $\mathrm{Br} \mathrm{J}$ Cancer 2015;112(3):580-93.

[21] Choi YJ, Myung SK, Lee JH. Light alcohol drinking and risk of cancer: a meta-analysis of cohort studies. Cancer Res Treat 2018;50(2):474-87.
[22] Dossus L, Boutron-Ruault MC, Kaaks R, et al. For the European Prospective Investigation into Cancer and (EPIC) nutrition cohort. Active and passive cigarette smoking and breast cancer risk: results from the EPIC cohort. Int J Cancer 2014;134(8):1871-88.

[23] Jones ME, Schoemaker MJ, Wright LB, et al. Smoking and risk of breast cancer in the Generations Study cohort. Breast Cancer Res 2017;19(1):118.

[24] Hardefeldt PJ, Penninkilampi R, Edirimanne S, et al. Physical activity and weight loss reduce the risk of breast cancer: a meta-analysis of 139 prospective and retrospective studies. Clin Breast Cancer 2018;18(4):e601-e12. 